\title{
Supporting Secure Deployment of Portal Components
}

\author{
Martin Gaedke, Johannes Meinecke, and Martin Nussbaumer \\ University of Karlsruhe, Institute of Telematics, \\ IT-Management and Web Engineering Research Group, \\ Engesserstr. 4, 76128 Karlsruhe, Germany \\ \{gaedke, meinecke, nussbaumer\}@tm. uni-karlsruhe.de
}

\begin{abstract}
With the growth of the World Wide Web, it has become more and more important to find implementation models tailored especially for Web applications. Unlike traditional applications, Web portals are required to be constantly up to date and are therefore often subject to changes at runtime. Consequently Web application frameworks have been developed that allow the construction and manipulation of Web pages consisting of pre-built components as an alternative to re-programming. The capability of such systems rises with the number of available components. Hence it is advisable to facilitate their deployment and exchange with a supporting architecture. The achieved increased use of third party components also results in higher risks for the provider of the portal. Especially the resource access by the contained code poses a potential threat and lowers the acceptance for foreign components. This paper proposes a robust architecture for the installation, administration and exchange of portal components and addresses the need for protection against malicious code.
\end{abstract}

\section{Introduction}

During the past years the World Wide Web has changed noticeably and is nowadays quickly moving forward towards a platform for distributed applications and services. Individual Web pages are typically aggregated to Web portals. Many elements of these portals are not specific to a single site and should therefore be subject to reuse. Component-Based Software Engineering (CBSE) [1] offers an answer to that problem by delegating functionality to separately exchangeable software artifacts. Consequently, CBSE strategies have been adopted for the development and evolution of Web applications [2], which is commonly denoted by the term Component-Based Web Engineering (CBWE).

Another concept for distributing Web application functionality is the use of Web services. In contrast to components that are hosted at the owner's realm, using a Web service hosted by third party requires trust. In other words, functionality is provided by granting access to the service instead of deploying it. This raises concerns regarding matters of trust and security. A big software company, for example, will not be convinced to manage their accountancy with the help of a Web service, possibly provided by a direct competitor. It will be less reluctant to buy a component from the same vendor and use it on one of their own servers, allowing it to keep control of the sensitive accounting data. 
Another aspect of Web portals is the required degree of flexibility. The information and functionality they offer has to be adapted quite frequently [3], these changes should be made as simple as possible. The WebComposition approach forms a solid foundation by focusing on configuring solutions instead of re-programming. An implementation of that approach, the WebComposition Service Linking System (WSLS) [4], has been developed. It provides a framework and supporting runtime system to assemble Web sites by using and configuring components.

In order to improve the degree of reuse, the components have to be transported between component-exchanging portals. This paper focuses on issues concerning the secure deployment, exchange and management of components for Web portals. We will investigate the current state of the art of according technologies and present an approach to deal with a selection of unsolved problems.

\section{State of the Art}

The application of CBSE concepts to the development of Web portals is not a new idea and has already been adopted by a range of systems:

A traditional strategy relies on the usage of software development environments like Microsoft Visual Studio .NET or the Sun ONE Application Framework. Graphical tools allow the construction of Web pages from components, with the possibility of integrating new components into the environment. The resulting applications have to be compiled and deployed on a Web server. After that, the formerly independent parts cannot be distinguished anymore, nor is it possible to insert new components or adjust existing ones without returning to the development phase.

Other solutions can be achieved by a second approach: resorting to componentbased portal frameworks like Microsoft SharePoint Services [5] and PHP-Nuke [6]. Comparably to content management systems, these portals enable administrators to edit their pages by using Web interfaces to insert and adjust interactive page components. In addition to a set of supplied components, the integration of independently developed software artifacts is supported. In many cases, the process of installing the new component files is not covered by a Web interface. Its complexity hinders widespread use of foreign components.

Another strategy for extendable portals is pursued by the use of Web Services. The Web Services for Remote Portlets (WSRP) [7] among others builds on this strategy by exporting reusable functionality including markup code generation into distant Web services. This makes the component installation process obsolete but poses other problems, as already mentioned.

The last regarded approach is the support for propagating components by publishing them with component repositories. Most corresponding systems focus on offering efficient means for searching the pool of components. A repository especially intended for CBWE is introduced in [2]. In this case, searching is supported by supplying extendable ways of representing and categorizing the contained components with arbitrary metadata. The technical retrieval in combination with the installation of a component into a portal is, however, left outside the concept. 


\section{Aspects of Deploying Components for Web Applications}

Given the state of the art, we designed a new architecture integrating component deployment with the exchange supported by a component repository. Special attention was paid to the reuse of third-party components with regard to protection against malicious code. Another investigated aspect not presented here due to limited space concerned the integration of licensing mechanisms.

In the context of Component-Based Web Engineering, we relate the term exchange to the complete course of events necessary for a component deployed on one Web portal to be transported and installed on another. We chose a distributed approach with the subjects of exchange stationed at the portal servers and a central directory serving as a registry for information about their properties and locations. Thus, this concept allows for applying the same infrastructure for the deployment of components as used for Web service discovery and integration.

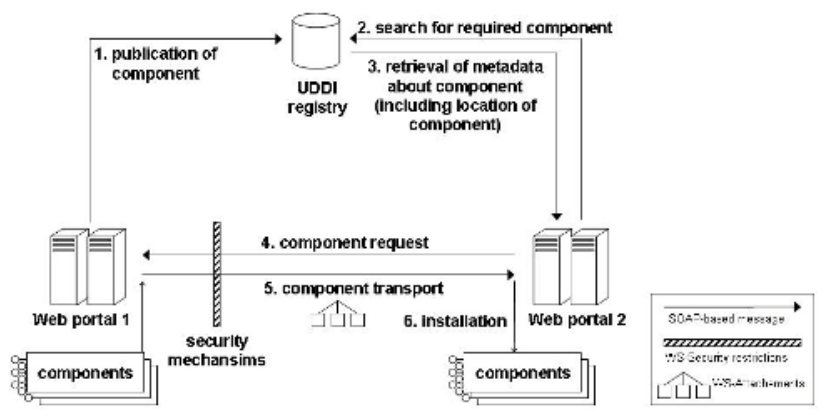

Fig. 1. Component Exchange Process

Figure 1 shows the individual steps of a component exchange between two portals. All activities are covered by supporting software, meaning that steps like contacting the UDDI registry are transparent for the portal administrators. To supplement the limited features of SOAP, some of the SOAP-based protocols of the Web Service Enhancements (WSE) [8] are used for the realization of the component request and transport. For example, the WS-Attachement protocol [9] allows sending large binary data sets (the component files) attached to the XML-encoded SOAP message. Also, WS-Security [10] provides the means for integrating security mechanisms into the inter-portal communication.

A problem still largely unsolved by the state of the art is the security risk that comes with the deployment of third-party portal components. Usually distributed in binary form, without additional mechanisms there is no way of monitoring or controlling their accesses on resources and data potentially worth of protection. Therefore, we integrated the concept of a robust code environment within the overall architecture.

Figure 2 contains an overview of a concept for a robust code environment. As a basic rule, code producers are to digitally sign their components. This prevents other parties from unnoticeably tampering with the binary code. It also helps to uniquely identify the authors to determine the set of rights issued to the component. During the installation process an early security check is performed. Among other aspects, the contained 
metadata is reviewed with respect to any statements about the behavior of the component. These could for example include declarations of resources the component needs to access, allowing the prevention of destabilizing problems at runtime. From the security perspective, this alone does not sufficiently protect the server, as the authors cannot be relied on correctly declaring all accesses. Therefore, additional security control at runtime restricts the installed code in its resource usage. Both mechanisms during installation and runtime are controlled by a flexible security policy differentiating between several levels of trust towards the component authors.

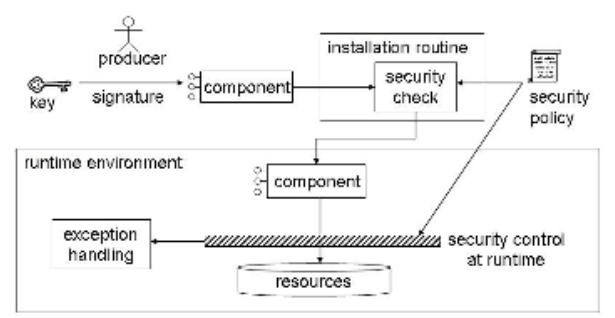

Fig. 2. Protection Mechanism against Foreign Code

\section{Pretoria System}

The Pretoria System implements the necessary support for the presented concepts. As the underlying technological platform for the component administration software and the component model, the Microsoft .NET Framework was chosen. Being a new member of the WebComposition approach, the Pretoria Administration Suite provides a secure infrastructure for component exchange. The screenshot in figure 3 shows a typical view of a component and some of its metadata. It allows administrators to install new components, manage existing ones and participate in the exchange process. The software is available for download at http://mw.tm.uni-karlsruhe.de/projects/pretoria.

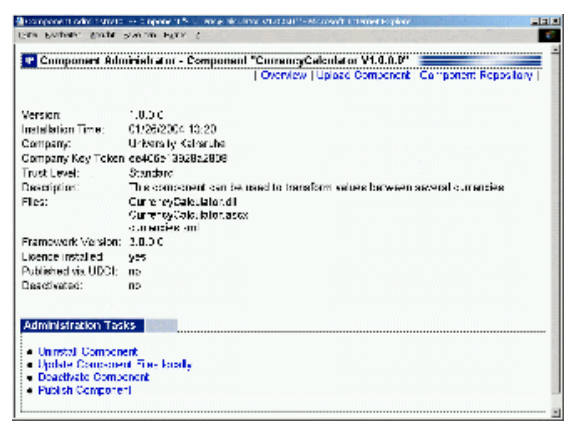

Fig. 3. View of an Installed Component within the Pretoria Administration Suite 


\section{Conclusion and Future Work}

In this paper, we discussed the deployment of components into Web portals and the support for their propagation through repositories. The evaluation of the state of the art raises the need for a system combining the exchange with the deployment process. Also the use of foreign code calls for additional resource protection. The problem can be solved with a distributed architecture making use of Web service technologies.

Plans for the future are to transform the presented architecture to other platforms like EJB or SharePoint, as it is independent of the underlying component model. Further plans are to integrate license mechanisms based on Digital Rights Management (DRM)

Acknowledgement. This material is based on research for the Mobile University (MUniversity) project supported by Microsoft Research Ltd and Microsoft Deutschland $\mathrm{GmbH}$.

\section{References}

1. Heineman, G. T., Councill, W. T.: Component-Based Software Engineering - Putting the Pieces Together. Addison-Wesley, Boston (2001)

2. Gaedke, M., Rehse, J.: Supporting Compositional Reuse in Component-Based Web Engineering. In Proceedings of the ACM Symposium on Applied Computing, ACM Press, (2000) 101-106

3. Koch, N., Rossi, G.: Patterns for Adaptive Web Applications. In $7^{\text {th }}$ European Conference on Pattern Languages of Programs, Germany (2002)

4. Gaedke, M., Nussbaumer, M.: The WebComposition Service Linking System (WSLS) project site. Germany (2002): http://mw.tm.uni-karlsruhe.de/projects/wsls/ (2/2004)

5. Microsoft: Microsoft SharePoint Products and Technologies - Web Site, USA (2004): http://www.microsoft.com/sharepoint/ (2/2004)

6. PHPNuke: PHPNuke Advanced Content Management System - Web Site (2004). http://phpnuke.org/ (2/2004)

7. Kropp, A., Leue, C., Thompson, R.: Web Services for Remote Portlets Specification Web Site, USA (2004): http://www.oasis-open.org/committees/wsrp/ (2/2004)

8. Powell, M.: Programming with Web Services Enhancements 2.0. MSDN, USA (2003): http://msdn.microsoft.com/webservices/building/wse/default.aspx (2/2004)

9. Barton, J. J., Thatte, S., Nielsen, H. F.: SOAP Messages with Attachments, W3C Note 11 December 2000, WWW Consortium, USA (2000), http://www.w3.org/TR/SOAP-attachments (2/2004)

10. Kaler, C., Hallam-Baker, P., Monzillo, R.: Web Services Security: SOAP Message Security. Working Draft 17, 27 August 2003, OASIS Web Site, USA (2003): http://www.oasis-open.org/committees/documents.php (2/2004) 